\title{
On a general $q$-identity
}

\author{
Aimin $\mathrm{Xu}^{*}$ \\ Institute of Mathematics \\ Zhejiang Wanli University \\ Ningbo 315100, China \\ xuaimin1009@hotmail.com; xuaimin@zwu.edu.cn
}

Submitted: Dec 12, 2013; Accepted: Apr 24, 2014; Published: May 9, 2014

Mathematics Subject Classifications: 05A19, 11B65

\begin{abstract}
In this paper, by means of the $q$-Rice formula we obtain a general $q$-identity which is a unified generalization of three kinds of identities. Some known results are special cases of ours. Meanwhile, some identities on $q$-generalized harmonic numbers are also derived.
\end{abstract}

Keywords: $q$-Rice formula; $q$-identity; $q$-generalized harmonic number; Cauchy's integral formula; Faà di Bruno's formula

\section{Introduction}

Three kinds of identities will be introduced in this paper.

In the paper [21], Van Hamme gave the following identity

$$
\sum_{k=1}^{n}(-1)^{k-1}\left[\begin{array}{l}
n \\
k
\end{array}\right]_{q} \frac{q^{\left(\begin{array}{c}
k+1 \\
2
\end{array}\right)}}{1-q^{k}}=\sum_{k=1}^{n} \frac{q^{k}}{1-q^{k}}
$$

One of the generalizations of (1.1) was given by Dilcher [6]:

$$
\sum_{k=1}^{n}(-1)^{k-1}\left[\begin{array}{l}
n \\
k
\end{array}\right]_{q} \frac{q^{\left(\begin{array}{c}
k \\
2
\end{array}\right)+k \lambda}}{\left(1-q^{k}\right)^{\lambda}}=\sum_{1 \leqslant \alpha_{1} \leqslant \cdots \leqslant \alpha_{\lambda} \leqslant n} \prod_{j=1}^{\lambda} \frac{q^{\alpha_{j}}}{1-q^{\alpha_{j}}}
$$

*Supported by the National Natural Science Foundation of China (grant 11201430), and the Ningbo Natural Science Foundation. 
Prodinger [16] gave another generalization of (1.1):

$$
\sum_{k=0, \neq m}^{n}(-1)^{k-1}\left[\begin{array}{l}
n \\
k
\end{array}\right] \frac{q_{q}^{\left(\begin{array}{c}
k+1 \\
2
\end{array}\right)}}{1-q^{k-m}}=(-1)^{m} q^{\left(\begin{array}{c}
m+1 \\
2
\end{array}\right)} \sum_{k=0, \neq m}^{n} \frac{q^{k}}{1-q^{k-m}},
$$

where $0 \leqslant m \leqslant n$. Many works have been devoted to the study of the generalizations of these identities. See for example [8, 9, 17, 23]. Recently, Guo and Zhang [12] made use of the Lagrange interpolation formula to give a generalization of Prodinger's identity (1.3). They also gave a generalization of Dilcher's identity (1.2). See Theorems 1.1 and 1.2 in [12], respectively. Ismail and Stanton used the theory of basic hypergeometric functions to generalize Dilcher's identity. See Theorem 2.2 in [13].

In the paper[5], Díaz-Barrero et al. obtained two identities involving rational sums:

$$
\begin{aligned}
\sum_{k=1}^{n}(-1)^{k-1}\left(\begin{array}{l}
n \\
k
\end{array}\right)\left(\begin{array}{c}
x+k \\
k
\end{array}\right)^{-1} \sum_{1 \leqslant \alpha \leqslant \beta \leqslant k} \frac{1}{x^{2}+(\alpha+\beta) x+\alpha \beta}=\frac{n}{(x+n)^{3}}, \\
\sum_{k=1}^{n}(-1)^{k-1}\left(\begin{array}{l}
n \\
k
\end{array}\right)\left(\begin{array}{c}
x+k \\
k
\end{array}\right)^{-1}\left\{\sum_{\alpha=1}^{k} \frac{1}{(x+\alpha)^{3}}+\sum_{1 \leqslant \alpha \leqslant \beta \leqslant k} \frac{1}{(x+\alpha)(x+\beta)(2 x+\alpha+\beta)}\right. \\
\left.+\sum_{1 \leqslant \alpha<\beta<\gamma \leqslant k} \frac{1}{(x+\alpha)(x+\beta)(x+\gamma)}\right\}=\frac{n}{(x+n)^{4}} .
\end{aligned}
$$

Recently, Prodinger [18] made use of partial fraction decomposition and inverse pairs to present a more general formula:

$$
\sum_{k=1}^{n}(-1)^{k-1}\left(\begin{array}{l}
n \\
k
\end{array}\right)\left(\begin{array}{c}
x+k \\
k
\end{array}\right)^{-1} \sum_{c_{1}+2 c_{2}+\cdots=\lambda} \prod_{j \geqslant 1} \frac{s_{k, j}^{c_{j}}}{c_{j} ! j^{c_{j}}}=\frac{n}{(x+n)^{\lambda+1}},
$$

where $s_{k, j}=\sum_{\alpha=1}^{k}(x+\alpha)^{-j}$. Almost at the same time, Chu and Yan [2] presented a generalization with multiple $\lambda$-fold sum:

$$
\sum_{k=0}^{n}(-1)^{k}\left(\begin{array}{c}
n \\
k
\end{array}\right)\left(\begin{array}{c}
x+k \\
k
\end{array}\right)^{-1} \sum_{0 \leqslant \alpha_{1} \leqslant \cdots \leqslant \alpha_{\lambda} \leqslant k} \prod_{j=1}^{\lambda} \frac{1}{x+\alpha_{j}}=\frac{x}{(x+n)^{\lambda+1}} .
$$

A direct proof of (1.5) can be found in Chu [1]. More recently, Mansour et al. [15] established a $q$-analog for the rational sum identity (1.4):

$$
\sum_{k=1}^{n}(-1)^{k-1} q^{\left(\begin{array}{c}
k \\
2
\end{array}\right)-k(n-1)}\left[\begin{array}{c}
n \\
k
\end{array}\right]_{q}\left[\begin{array}{c}
x+k \\
k
\end{array}\right]_{q}^{-1} \sum_{c_{1}+2 c_{2}+\cdots=\lambda} \prod_{j \geqslant 1} \frac{s_{k, j}(q)^{c_{j}}}{c_{j} ! j^{c_{j}}}=\frac{q^{n \lambda}[n]_{q}}{[x+n]_{q}^{\lambda+1}}
$$

where $s_{k, j}(q)=\sum_{\alpha=1}^{k} q^{j \alpha}[x+\alpha]_{q}^{-j}$. In particular, they gave a very nice bijective proof for the case $\lambda=1$. 
In the recent paper [19], Prodinger established an interesting identity involving harmonic numbers:

$$
\begin{aligned}
& \sum_{k=0, \neq m}^{n}(-1)^{k-1}\left(\begin{array}{l}
n \\
k
\end{array}\right)\left(\begin{array}{c}
n+k \\
k
\end{array}\right) \frac{1}{(k-m)^{\lambda}} \\
& \quad=(-1)^{m}\left(\begin{array}{c}
n \\
m
\end{array}\right)\left(\begin{array}{c}
n+m \\
n
\end{array}\right) \sum_{c_{1}+2 c_{2}+\cdots=\lambda} \frac{1}{c_{1} ! c_{2} ! \cdots} \prod_{j=1}^{\lambda}\left(\frac{\mathcal{H}_{j}}{j}\right)^{c_{j}},
\end{aligned}
$$

where

$$
\mathcal{H}_{j}=(-1)^{j-1}\left(H_{m+n}^{(j)}-2 H_{m}^{(j)}\right)+H_{n-m}^{(j)},
$$

and $H_{n}^{(r)}$ are the generalized harmonic numbers defined by

$$
H_{0}^{(r)}=0, \quad H_{n}^{(r)}=\sum_{k=1}^{n} \frac{1}{k^{r}} \quad \text { for } \quad n, r=1,2, \ldots
$$

Mansour [14] obtained a general rational sum to generalize this identity. He also obtained a $q$-analog of this result involving $q$-harmonic numbers.

Motivated by these interesting work, by means of the $q$-Rice formula used in $[16,17]$, we will establish a general $q$-identity which is a common generalization of those three kinds of identities introduced before.

Theorem 1.1. Let $\lambda$ be any positive integer. For $0 \leqslant m \leqslant n$ and $0 \leqslant l \leqslant n+\lambda-1$, there holds

$$
\begin{aligned}
& \sum_{k=0, \neq m}^{n}\left[\begin{array}{l}
n \\
k
\end{array}\right]_{q} \frac{q^{(\lambda-1) k+m}\left(1-q^{k-m}\right)(q / z ; q)_{k}\left(z q^{-l} ; q\right)_{n-k+\lambda-1}}{\left(1-x q^{k-m}\right)^{\lambda+1}} z^{k} \\
& =-\frac{(q ; q)_{n}\left(z q^{-l} ; q\right)_{l}\left(z x q^{-m} ; q\right)_{n-l+\lambda-1}}{\left(x q^{-m} ; q\right)_{m}(x q ; q)_{n-m}} \sum_{\|\vec{c}\|=\lambda} \frac{1}{\bar{c} !} \prod_{j=1}^{\lambda}\left(\frac{u_{j}}{j}\right)^{c_{j}}
\end{aligned}
$$

where $\vec{c} !=c_{1} ! c_{2} ! \cdots c_{\lambda} !,\|\vec{c}\|=c_{1}+2 c_{2}+\cdots+\lambda c_{\lambda}$ and

$$
u_{j}=-\sum_{k=0}^{n-l+\lambda-2}\left(\frac{z q^{k}}{1-z x q^{k-m}}\right)^{j}+\sum_{k=0, \neq m}^{n}\left(\frac{q^{k}}{1-x q^{k-m}}\right)^{j} .
$$

This is a very general $q$-series sum identity involving five parameters $\lambda, l, m, x$ and $z$. It contains several known identities by choosing different parameters, which will be shown in the third section. By means of our identity, we will also obtain some identities on $q$-generalized harmonic numbers.

Throughout this paper, we will use the standard notation. For any real number $x$ and any integer $m$, define

$$
[x]_{q}=\frac{1-q^{x}}{1-q}, \quad(x ; q)_{\infty}=\prod_{k=0}^{\infty}\left(1-x q^{k}\right), \quad(x ; q)_{m}=\frac{(x ; q)_{\infty}}{\left(x q^{m} ; q\right)_{\infty}} .
$$


For any nonnegative integer $n$, define

$$
[n]_{q} !=[1]_{q}[2]_{q} \cdots[n]_{q},\left[\begin{array}{l}
n \\
k
\end{array}\right]_{q}=\frac{[n]_{q} !}{[k]_{q} ![n-k]_{q} !} .
$$

\section{Proof of Theorem 1.1}

In the very interesting paper [16], Prodinger introduced the following formula

$$
\sum_{k=1}^{n}(-1)^{k-1} q^{\left(\begin{array}{c}
k \\
2
\end{array}\right)}\left[\begin{array}{l}
n \\
k
\end{array}\right]_{q} f\left(q^{-k}\right)=\frac{1}{2 \pi i} \int_{\mathcal{C}} \frac{(q ; q)_{n}}{(t ; q)_{n+1}} f(t) d t
$$

where $\mathcal{C}$ encircles the poles $q^{-1}, q^{-2}, \ldots, q^{-n}$ and no other. It is a $q$-analog of Rice's formula $[7,20]$ :

$$
\sum_{k=1}^{n}\left(\begin{array}{l}
n \\
k
\end{array}\right)(-1)^{k} f(k)=\frac{(-1)^{n}}{2 \pi i} \int_{\mathcal{C}} \frac{n !}{t(t-1) \cdots(t-n)} f(t) d t,
$$

where $\mathcal{C}$ encircles the poles $1,2, \ldots, n$ and no other. Indeed, by Cauchy's integral formula one is not hard to find that for any integer $m \in\{0,1, \ldots, n\}$ there holds

$$
\sum_{k=0, \neq m}^{n}(-1)^{k-1} q^{\left(\begin{array}{c}
k \\
2
\end{array}\right)}\left[\begin{array}{l}
n \\
k
\end{array}\right]_{q} f\left(q^{-k}\right)=(-1)^{n-1} \frac{(q ; q)_{n}}{q^{\left(\begin{array}{c}
n+1 \\
2
\end{array}\right)}} \frac{1}{2 \pi i} \int_{\mathcal{C}} \frac{f(t) d t}{\prod_{j=0}^{n}\left(t-q^{-j}\right)}
$$

where $\mathcal{C}$ encircles the poles $q^{-j}, j \in\{0,1, \ldots, n\}-\{m\}$ and no other. Prodinger first applied the $q$-analog of Rice's formula to prove many identities such as the identities of Van Hamme, Uchimura, Dilcher, Andrews-Crippa-Simon, and Fu-Lascoux, see [16, 17] and references therein. It was shown that this formula is a very powerful and useful tool. Now, in this section we will use this important formula and present a proof of Theorem 1.1.

Proof of Theorem 1.1. By simple calculations we have

$$
\begin{aligned}
& \sum_{k=0, \neq m}^{n}\left[\begin{array}{l}
n \\
k
\end{array}\right]_{q} \frac{q^{(\lambda-1) k}\left(1-q^{k-m}\right)(q / z ; q)_{k}\left(z q^{-l} ; q\right)_{n-k+\lambda-1}}{\left(1-x q^{k-m}\right)^{\lambda+1}} z^{k} \\
= & \sum_{k=0, \neq m}^{n}(-1)^{k} q^{\left(\begin{array}{l}
k \\
2
\end{array}\right)+k \lambda}\left[\begin{array}{l}
n \\
k
\end{array}\right]_{q} \frac{1-q^{k-m}}{\left(1-x q^{k-m}\right)^{\lambda+1}}\left(z q^{-k} ; q\right)_{k}\left(z q^{-l} ; q\right)_{n-k+\lambda-1} \\
= & \sum_{k=0, \neq m}^{n}(-1)^{k} q^{\left(\begin{array}{c}
k \\
2
\end{array}\right)+k \lambda}\left[\begin{array}{l}
n \\
k
\end{array}\right]_{q} \frac{1-q^{k-m}}{\left(1-x q^{k-m}\right)^{\lambda+1}} \frac{\left(z q^{-k} ; q\right)_{\infty}}{(z ; q)_{\infty}} \frac{\left(z q^{-l} ; q\right)_{\infty}}{\left(z q^{n+\lambda-1-l-k} ; q\right)_{\infty}} \\
= & \left(z q^{-l} ; q\right)_{l} \sum_{k=0, \neq m}^{n}(-1)^{k} q^{\left(\begin{array}{c}
k \\
2
\end{array}\right)+k \lambda}\left[\begin{array}{c}
n \\
k
\end{array}\right]_{q} \frac{1-q^{k-m}}{\left(1-x q^{k-m}\right)^{\lambda+1}}\left(z q^{-k} ; q\right)_{n-l+\lambda-1} .
\end{aligned}
$$


Thus, by the $q$-Rice formula (2.1) there holds

$$
\begin{aligned}
& \sum_{k=0, \neq m}^{n}\left[\begin{array}{l}
n \\
k
\end{array}\right]_{q} \frac{q^{(\lambda-1) k}\left(1-q^{k-m}\right)(q / z ; q)_{k}\left(z q^{-l} ; q\right)_{n-k+\lambda-1}}{\left(1-x q^{k-m}\right)^{\lambda+1}} z^{k} \\
= & (-1)^{n}\left(z q^{-l} ; q\right)_{l} \frac{(q ; q)_{n}}{q^{\left(\begin{array}{c}
n+1 \\
2
\end{array}\right)} \frac{1}{2 \pi i}} \int_{\mathcal{C}} \frac{(z t ; q)_{n-l+\lambda-1} d t}{\left(t-x q^{-m}\right)^{\lambda+1} \prod_{k=0, \neq m}^{n}\left(t-q^{-k}\right)},
\end{aligned}
$$

where $\mathcal{C}$ (positively oriented) encloses the poles $q^{-j}, j \in\{0,1, \ldots, n\}-\{m\}$ and no other. It is obvious that

$$
\frac{1}{2 \pi i} \int_{\mathcal{C}} \frac{(z t ; q)_{n-l+\lambda-1} d t}{\left(t-x q^{-m}\right)^{\lambda+1} \prod_{k=0, \neq m}^{n}\left(t-q^{-k}\right)}=-\frac{1}{2 \pi i} \int_{\mathcal{C}^{\prime}} \frac{(z t ; q)_{n-l+\lambda-1} d t}{\left(t-x q^{-m}\right)^{\lambda+1} \prod_{k=0, \neq m}^{n}\left(t-q^{-k}\right)}
$$

where $\mathcal{C}^{\prime}$ (positively oriented) encloses the pole $x q^{-m}$. By Cauchy's integral formula, there holds

$$
\frac{1}{2 \pi i} \int_{\mathcal{C}^{\prime}} \frac{(z t ; q)_{n-l+\lambda-1}}{\left(t-x q^{-m}\right)^{\lambda+1} \prod_{k=0, \neq m}^{n}\left(t-q^{-k}\right)}=\left.\frac{1}{\lambda !} \frac{d^{\lambda}}{d t^{\lambda}} \frac{(z t ; q)_{n-l+\lambda-1}}{\prod_{k=0, \neq m}^{n}\left(t-q^{-k}\right)}\right|_{t=x q^{-m}} .
$$

Applying Faà di Bruno's formula [4] yields

$$
\begin{aligned}
\left.\frac{d^{\lambda}}{d t^{\lambda}} \frac{(z t ; q)_{n-l+\lambda-1}}{\prod_{k=0, \neq m}^{n}\left(t-q^{-k}\right)}\right|_{t=x q^{-m}} & =\left.\frac{d^{\lambda}}{d t^{\lambda}} e^{\sum_{k=0}^{n-l+\lambda-2} \log \left(1-z t q^{k}\right)-\sum_{k=0, \neq m}^{n} \log \left(t-q^{-k}\right)}\right|_{t=x q^{-m}} \\
& =\frac{\left(z x q^{-m} ; q\right)_{n-l+\lambda-1}}{\prod_{k=0, \neq m}^{n}\left(x q^{-m}-q^{-k}\right)} \sum_{\|\vec{c}\|=\lambda} \frac{1}{\vec{c} !} \prod_{j=1}^{\lambda}\left(\frac{u_{j}}{j}\right)^{c_{j}} .
\end{aligned}
$$

From (2.2), (2.3),(2.4) and (2.5), the desired result is obtained.

Remark 2.1. Actually, careful checking the proof of Theorem 1.1, one can find that Theorem 1.1 still holds for $\lambda=0$ if in this case we assume the sum of the right hand side of (1.8) is equal to 1 . This implies that for $0 \leqslant l \leqslant n-1$ there holds

$$
\sum_{k=0, \neq m}^{n}\left[\begin{array}{l}
n \\
k
\end{array}\right]_{q} \frac{\left(1-q^{m-k}\right)(q / z ; q)_{k}\left(z q^{-l} ; q\right)_{n-k-1}}{1-x q^{k-m}} z^{k}=\frac{(q ; q)_{n}\left(z q^{-l} ; q\right)_{l}\left(z x q^{-m} ; q\right)_{n-l-1}}{\left(x q^{-m} ; q\right)_{m}(x q ; q)_{n-m}} .
$$

\section{Consequences of Theorem 1.1}

Theorem 1.1 can help us to find some new identities or retrieve some well known identities.

Let $\lambda=1$ and $x=1$. (1.8) reduces to the following identity. 
Corollary 3.1. For $0 \leqslant m \leqslant n$ and $0 \leqslant l \leqslant n$, there holds

$$
\begin{aligned}
& \sum_{k=0, \neq m}^{n}\left[\begin{array}{l}
n \\
k
\end{array}\right]_{q} \frac{(q / z ; q)_{k}\left(z q^{-l} ; q\right)_{n-k}}{1-q^{k-m}} z^{k} \\
& =(-1)^{m-1} q^{\left(\begin{array}{c}
m \\
2
\end{array}\right)}\left[\begin{array}{c}
n \\
m
\end{array}\right]_{q}\left(z q^{-l} ; q\right)_{l}\left(z q^{-m} ; q\right)_{n-l}\left\{-\sum_{k=0}^{n-l-1} \frac{z q^{k}}{1-z q^{k-m}}+\sum_{k=0, \neq m}^{n} \frac{q^{k}}{1-q^{k-m}}\right\} \text {. }
\end{aligned}
$$

Guo and Zhang [12] made use of Lagrange interpolation formula to obtain this identity which generalizes the identity (1.3) due to Prodinger. It is obvious that (3.1) reduces to (1.3) when $l=0$ and $z \rightarrow 0$.

Let $x=1, l=\lambda-1$ and $z=q^{-n}$ in (1.8). We have

Corollary 3.2. Let $\lambda$ be any nonnegative integer. For $0 \leqslant m \leqslant n$, there holds

$$
\begin{aligned}
& \sum_{k=0, \neq m}^{n}(-1)^{k-1}\left[\begin{array}{l}
n \\
k
\end{array}\right]_{q}\left[\begin{array}{c}
n+k \\
k
\end{array}\right]_{q} \frac{q^{\left(\begin{array}{c}
k \\
2
\end{array}\right)+(\lambda-n) k}}{\left(1-q^{k-m}\right)^{\lambda}} \\
& =(-1)^{m} q^{\left(\begin{array}{c}
m \\
2
\end{array}\right)-n m}\left[\begin{array}{l}
n \\
m
\end{array}\right]_{q}\left[\begin{array}{c}
n+m \\
n
\end{array}\right]_{q} \sum_{\|\vec{c}\|=\lambda} \frac{1}{\vec{c} !} \prod_{j=1}^{\lambda}\left(\frac{\mathcal{H}_{j}(q)}{j}\right)^{c_{j}},
\end{aligned}
$$

where

$$
\mathcal{H}_{j}(q)=-\sum_{k=0}^{n-1}\left(\frac{q^{k-n}}{1-q^{k-m-n}}\right)^{j}+\sum_{k=0, \neq m}^{n}\left(\frac{q^{k}}{1-q^{k-m}}\right)^{j} .
$$

This identity is a $q$-analog of Prodinger's identity (1.7). An alternative form of this $q$-identity was presented in [14].

For $m=0, l=0$ and $z \rightarrow 0$ in (1.8), the following identity is true.

Corollary 3.3. Let $\lambda$ be any nonnegative integer. There holds

$$
\sum_{k=1}^{n}\left[\begin{array}{l}
n \\
k
\end{array}\right]_{q}(-1)^{k-1} q^{\left(\begin{array}{c}
k \\
2
\end{array}\right)+\lambda k} \frac{1-q^{k}}{\left(1-x q^{k}\right)^{\lambda+1}}=\frac{(q ; q)_{n}}{(x q ; q)_{n}} \sum_{\|\vec{c}\|=\lambda} \frac{1}{\bar{c} !} \prod_{j=1}^{\lambda}\left(\frac{1}{j} \sum_{k=1}^{n} \frac{q^{j k}}{\left(1-x q^{k}\right)^{j}}\right)^{c_{j}} .
$$

It is clear that

$$
\prod_{j=1}^{n} \frac{1}{1-x_{j} t}=\prod_{j=1}^{n} \sum_{k \geqslant 0}\left(x_{j} t\right)^{k}=\sum_{\lambda \geqslant 0} t^{\lambda} \sum_{1 \leqslant \alpha_{1} \leqslant \cdots \leqslant \alpha_{\lambda} \leqslant n} \prod_{j=1}^{\lambda} x_{\alpha_{j}} .
$$

Since

$$
\left.\frac{d^{\lambda}}{d t^{\lambda}} \prod_{j=1}^{n} \frac{1}{1-x_{j} t}\right|_{t=0}=\left.\frac{d^{\lambda}}{d t^{\lambda}} e^{-\sum_{j=1}^{n} \log \left(1-x_{j} t\right)}\right|_{t=0}
$$


we apply Faà di Bruno's formula [4] to obtain

$$
\left.\frac{d^{\lambda}}{d t^{\lambda}} \prod_{j=1}^{n} \frac{1}{1-x_{j} t}\right|_{t=0}=\sum_{\|\vec{c}\|=\lambda} \frac{\lambda !}{\vec{c} !} \prod_{j=1}^{\lambda}\left(\frac{\sum_{k=1}^{n} x_{k}^{j}}{j}\right)^{c_{j}} .
$$

Comparing (3.3) with (3.4), there holds

$$
\sum_{1 \leqslant \alpha_{1} \leqslant \cdots \leqslant \alpha_{\lambda} \leqslant n} \prod_{j=1}^{\lambda} x_{\alpha_{j}}=\sum_{\|\vec{c}\|=\lambda} \frac{1}{\vec{c} !} \prod_{j=1}^{\lambda}\left(\frac{\sum_{k=1}^{n} x_{k}^{j}}{j}\right)^{c_{j}} .
$$

Therefore, (3.2) can be rewritten as

$$
\sum_{k=1}^{n}\left[\begin{array}{l}
n \\
k
\end{array}\right]_{q}(-1)^{k-1} q^{\left(\begin{array}{c}
k \\
2
\end{array}\right)+\lambda k} \frac{1-q^{k}}{\left(1-x q^{k}\right)^{\lambda+1}}=\frac{(q ; q)_{n}}{(x q ; q)_{n}} \sum_{1 \leqslant \alpha_{1} \leqslant \ldots \leqslant \alpha_{\lambda} \leqslant n} \prod_{j=1}^{\lambda} \frac{q^{\alpha_{j}}}{1-x q^{\alpha_{j}}},
$$

or

$$
\sum_{k=1}^{n}\left[\begin{array}{l}
n \\
k
\end{array}\right]_{q}(-1)^{k-1} q^{\left(\begin{array}{l}
k \\
2
\end{array}\right)+\lambda k} \frac{1-q^{k}}{\left(1-x q^{k}\right)^{\lambda+1}}=\frac{(q ; q)_{n}}{(x q ; q)_{n}} \sum_{|\vec{b}|=\lambda} \prod_{j=1}^{n}\left(\frac{q^{j}}{1-x q^{j}}\right)^{b_{j}}
$$

where $|\vec{b}|=b_{1}+b_{2}+\cdots+b_{n}$. By the theory of basic hypergeometric functions Ismail and Stanton [13] found Eq. (3.5) which reduces to the Dilcher identity [6] when $x=1$.

In fact, it has been recently pointed out in [11] that the Ismail-Stanton result (3.5) is the $i=1$ (with $m=\lambda+1$ ) case of following formula due to Zeng [23]:

$$
\sum_{k=i}^{n}(-1)^{k-i}\left[\begin{array}{l}
n \\
k
\end{array}\right]_{q}\left[\begin{array}{l}
k \\
i
\end{array}\right]_{q} \frac{q^{\left(\begin{array}{c}
k-i \\
2
\end{array}\right)+k m}}{\left(1-z q^{k}\right)^{m}}=\frac{q^{i}(q ; q)_{i-1}(q ; q)_{n}}{(q ; q)_{i}(z q ; q)_{n}} h_{m-1}\left(\frac{q^{i}}{1-z q^{i}}, \ldots, \frac{q^{n}}{1-z q^{n}}\right),
$$

where $1 \leqslant i \leqslant n$ and $h_{k}\left(x_{1}, \ldots, x_{n}\right)$ is the $k$ th homogeneous symmetric polynomial in $x_{1}, x_{2}, \ldots, x_{n}$ defined by

$$
h_{k}\left(x_{1}, \ldots, x_{n}\right)=\sum_{1 \leqslant i_{1} \leqslant \cdots \leqslant i_{k} \leqslant n} x_{i_{1}} \cdots x_{i_{k}}=\sum_{|\vec{b}|=k} x_{1}^{b_{1}} \cdots x_{n}^{b_{n}} .
$$

This more general formula can not follow from Theorem 1.1 and it can be viewed as a different generalization of the Ismail-Stanton result (3.5).

Since

$$
\left[\begin{array}{l}
n \\
k
\end{array}\right]_{q}=\left[\begin{array}{l}
n-1 \\
k-1
\end{array}\right]_{q} \frac{1-q^{n}}{1-q^{k}}
$$

Eq. (3.2) can be rewritten as

$$
\sum_{k=1}^{n}(-1)^{k-1}\left[\begin{array}{l}
n-1 \\
k-1
\end{array}\right]_{q} q^{\left(\begin{array}{c}
k \\
2
\end{array}\right)+\lambda k} \frac{1}{\left(1-x q^{k}\right)^{\lambda+1}}=\frac{(q ; q)_{n-1}}{(x q ; q)_{n}} \sum_{\|\vec{c}\|=\lambda} \frac{1}{\vec{c} !} \prod_{j=1}^{\lambda}\left(\frac{1}{j} \sum_{\alpha=1}^{n} \frac{q^{j \alpha}}{\left(1-x q^{\alpha}\right)^{j}}\right)^{c_{j}} .
$$


Using the $q$-inverse pair formula [10]

$$
f_{n}=\sum_{k=1}^{n}(-1)^{k} q^{\left(\begin{array}{c}
k \\
2
\end{array}\right)}\left[\begin{array}{l}
n-1 \\
k-1
\end{array}\right]_{q} g_{k} \Leftrightarrow g_{n}=\sum_{k=1}^{n}(-1)^{k} q^{\left(\begin{array}{c}
k \\
2
\end{array}\right)-k(n-1)}\left[\begin{array}{l}
n-1 \\
k-1
\end{array}\right]_{q} f_{k}
$$

we obtain the inverse of $(3.2)$

$$
\begin{aligned}
& \sum_{k=1}^{n}(-1)^{k-1} q^{\left(\begin{array}{l}
k \\
2
\end{array}\right)-k(n-1)}\left[\begin{array}{l}
n-1 \\
k-1
\end{array}\right]_{q} \frac{(q ; q)_{k-1}}{(x q ; q)_{k}} \\
& \times \sum_{\|\vec{c}\|=\lambda} \frac{1}{\vec{c} !} \prod_{j=1}^{\lambda}\left(\frac{1}{j} \sum_{\alpha=1}^{k} \frac{q^{j \alpha}}{\left(1-x q^{\alpha}\right)^{j}}\right)^{c_{j}}=\frac{q^{n \lambda}}{\left(1-x q^{n}\right)^{\lambda+1}} .
\end{aligned}
$$

Replacing $x$ by $q^{x}$, we rediscover an identity due to Mansour et al. [15]:

Corollary 3.4. Let $\lambda$ be any nonnegative integer. There holds

$$
\begin{aligned}
\sum_{k=1}^{n}(-1)^{k-1} q^{\left(\begin{array}{l}
k \\
2
\end{array}\right)-k(n-1)}\left[\begin{array}{l}
n \\
k
\end{array}\right]_{q}\left[\begin{array}{c}
x+k \\
k
\end{array}\right]_{q}^{-1} \\
\times \sum_{\|\vec{c}\|=\lambda} \frac{1}{\vec{c} !} \prod_{j=1}^{\lambda}\left(\frac{1}{j} \sum_{\alpha=1}^{k} \frac{q^{j \alpha}}{[x+\alpha]_{q}^{j}}\right)^{c_{j}}=\frac{q^{n \lambda}[n]_{q}}{[x+n]_{q}^{\lambda+1}} .
\end{aligned}
$$

This identity is a $q$-analog for the rational sum identity (1.4) due to Prodinger. If we further replace $n$ by $n+1$ and $x$ by $x-1$ in (3.6), then a $q$-analog of Chu-Yan's identity (1.5) is derived:

Corollary 3.5. Let $\lambda$ be any nonnegative integer. There holds

$$
\sum_{k=0}^{n}(-1)^{k} q^{\left(\begin{array}{c}
k+1 \\
2
\end{array}\right)-k n}\left[\begin{array}{l}
n \\
k
\end{array}\right]_{q}\left[\begin{array}{c}
x+k \\
k
\end{array}\right]_{q}^{-1} \sum_{0 \leqslant \alpha_{1} \leqslant \cdots \leqslant \alpha_{\lambda} \leqslant k} \prod_{j=1}^{\lambda} \frac{q^{\alpha_{j}}}{\left[x+\alpha_{j}\right]_{q}}=\frac{q^{n(\lambda+1)}[x]_{q}}{[x+n]_{q}^{\lambda+1}} .
$$

Let the generalized $q$-harmonic numbers

$$
H_{0}^{(r)}(q)=0, \quad H_{n}^{(r)}(q)=\sum_{k=1}^{n} q^{r k}[k]^{-r}, \quad n \geqslant 1 .
$$

Recently, the $q$-generalized harmonic number sums have been useful in studying Feynman diagram contributions an relations among special functions [3]. Taking $x=0$ in (3.6), we have the following identities on $q$-generalized harmonic numbers:

Corollary 3.6. For $\lambda \geqslant 1$, there holds

$$
\sum_{k=0}^{n}(-1)^{k} q^{\left(\begin{array}{c}
k+1 \\
2
\end{array}\right)-(k+\lambda) n}\left[\begin{array}{l}
n \\
k
\end{array}\right] \sum_{q\|\vec{c}\|=\lambda} \frac{1}{\bar{c} !} \prod_{j=1}^{\lambda}\left(\frac{H_{k}^{(j)}(q)}{j}\right)^{c_{j}}=-\frac{1}{[n]_{q}^{\lambda}} .
$$


The first few cases are listed as follows.

$$
\begin{aligned}
& \sum_{k=0}^{n}(-1)^{k} q^{\left(\begin{array}{c}
k+1 \\
2
\end{array}\right)-(k+1) n}\left[\begin{array}{l}
n \\
k
\end{array}\right]_{q} H_{k}(q)=-\frac{1}{[n]_{q}}, \\
& \sum_{k=0}^{n}(-1)^{k} q^{\left(\begin{array}{c}
k+1 \\
2
\end{array}\right)-(k+2) n}\left[\begin{array}{l}
n \\
k
\end{array}\right]_{q}\left(\left(H_{k}(q)\right)^{2}+H_{k}^{(2)}(q)\right)=-\frac{2}{[n]_{q}^{2}}, \\
& \sum_{k=0}^{n}(-1)^{k} q^{\left(\begin{array}{c}
k+1 \\
2
\end{array}\right)-(k+3) n}\left[\begin{array}{l}
n \\
k
\end{array}\right]_{q}\left(\left(H_{k}(q)\right)^{3}+3 H_{k}(q) H_{k}^{(2)}(q)+2 H_{k}^{(3)}(q)\right)=-\frac{6}{[n]_{q}^{3}}, \\
& \sum_{k=0}^{n}(-1)^{k} q^{\left(\begin{array}{c}
k+1 \\
2
\end{array}\right)-(k+4) n}\left[\begin{array}{l}
n \\
k
\end{array}\right]_{q}\left(\left(H_{k}(q)\right)^{4}+6\left(H_{k}(q)\right)^{2} H_{k}^{(2)}(q)+3\left(H_{k}^{(2)}(q)\right)^{2}\right. \\
& \left.\quad+8 H_{k}(q) H_{k}^{(3)}(q)+6 H_{k}^{(4)}(q)\right)=-\frac{24}{[n]_{q}^{4}}, \\
& \sum_{k=0}^{n}(-1)^{k} q^{\left(\begin{array}{c}
k+1 \\
2
\end{array}\right)-(k+5) n}\left[\begin{array}{l}
n \\
k
\end{array}\right]_{q}\left(\left(H_{k}(q)\right)^{5}+10\left(H_{k}(q)\right)^{3} H_{k}^{(2)}(q)+15 H_{k}(q)\left(H_{k}^{(2)}(q)\right)^{2}\right. \\
& \left.+20\left(H_{k}(q)\right)^{2} H_{k}^{(3)}(q)+20 H_{k}^{(2)}(q) H_{k}^{(3)}(q)+30 H_{k}(q) H_{k}^{(4)}(q)+24 H_{k}^{(5)}(q)\right)=-\frac{120}{[n]_{q}^{5}}
\end{aligned}
$$

These identities are $q$-analogs of generalized harmonic number identities which were presented in [22]:

$$
\begin{aligned}
& \sum_{k=0}^{n}(-1)^{k}\left(\begin{array}{l}
n \\
k
\end{array}\right) H_{k}=-\frac{1}{n}, \\
& \sum_{k=0}^{n}(-1)^{k}\left(\begin{array}{l}
n \\
k
\end{array}\right)\left(H_{k}^{2}+H_{k}^{(2)}\right)=-\frac{2}{n^{2}}, \\
& \sum_{k=0}^{n}(-1)^{k}\left(\begin{array}{l}
n \\
k
\end{array}\right)\left(H_{k}^{3}+3 H_{k} H_{k}^{(2)}+2 H_{k}^{(3)}\right)=-\frac{6}{n^{3}}, \\
& \sum_{k=0}^{n}(-1)^{k}\left(\begin{array}{l}
n \\
k
\end{array}\right)\left(H_{k}^{4}+6 H_{k}^{2} H_{k}^{(2)}+3\left(H_{k}^{(2)}\right)^{2}+8 H_{k} H_{k}^{(3)}+6 H_{k}^{(4)}\right)=-\frac{24}{n^{4}}, \\
& \sum_{k=0}^{n}(-1)^{k}\left(\begin{array}{l}
n \\
k
\end{array}\right)\left(H_{k}^{5}+10 H_{k}^{3} H_{k}^{(2)}+15 H_{k}\left(H_{k}^{(2)}\right)^{2}\right. \\
& \left.\quad+20 H_{k}^{2} H_{k}^{(3)}+20 H_{k}^{(2)} H_{k}^{(3)}+30 H_{k} H_{k}^{(4)}+24 H_{k}^{(5)}\right)=-\frac{120}{n^{5}}
\end{aligned}
$$

It is worth noticing that starting from

$$
\sum_{k=0}^{n}(-1)^{k} q^{\left(\begin{array}{c}
k+1 \\
2
\end{array}\right)-k n}\left[\begin{array}{l}
n \\
k
\end{array}\right]_{q} \frac{(q ; q)_{k}}{(x q ; q)_{k}}=\frac{q^{n}(1-x)}{1-x q^{n}}
$$


and taking the $j$ th derivative of both sides at $x=1$, we can also arrive at Corollary 3.6. Wang and Jia [22] applied the Newton-Andrews method to some well known identities and found many interesting identities on harmonic numbers which include the identities from (3.7) to (3.11).

\section{Acknowledgements}

We thank the anonymous referee for his/her careful reading of our manuscript and very helpful comments.

\section{References}

[1] W. Chu. Summation formulae involving harmonic numbers. Filomat, 26(1):143-152, 2012.

[2] W. Chu and Q.L. Yan. Combinatorial identities on binomial coefficients and harmonic numbers. Utilitas Mathematica, 75:51-66, 2008.

[3] M.W. Coffey. On a three-dimensional symmetric Ising tetrahedron, and contribitions to the theory of the dilogarithm and Clausen functions. J. Math. Phys., 49:0435101-043510-32, 2008.

[4] L. Comtet. Advanced combinatorics, the art of finite and infinite expansions. D. Reidel Publishing Co., Dordrecht, 1974.

[5] J.L. Díaz-Barrero, J. Gibergans-Báguena, and P.G. Popescu. Some identities involving rational sums. Appl. Anal. Discrete Math., 1:397-402, 2007.

[6] K. Dilcher. Some $q$-series identities related to divisor function. Discrete Math., 145(1-3):83-93, 1995.

[7] P. Flajolet and R. Sedgewick. Mellin transforms and asymptotics: Finite differences and Rice's integrals. Theoretical Computer Sci., 144:101-124, 1995.

[8] A.M. Fu and A. Lascoux. q-Identities from Lagrange and Newton interpolation. Adv. Appl. Math., 31:527-531, 2003.

[9] A.M. Fu and A. Lascoux. q-Identities related to overpartitions and divisor functions. Electron. J. Combin., 12:\#R38, 2005.

[10] I.P. Goulden and D.M. Jackson. Combinatorial enumeration. A Wiley-Interscience Publication, John Wiley \& Sons Inc., New York, 1983. (With a foreword by GianCarlo Rota).

[11] V.J.W. Guo and J. Zeng. Further $(p, q)$-identities related to divisor functions. arXiv:1312.6537

[12] V.J.W. Guo and C. Zhang. Some further $q$-series identities related to divisor functions. Ramanujan J., 25(3):295-306, 2011.

[13] M.E.H. Ismail and D. Stanton. Some combinatorial and analytical identities. Ann. Comb., 16:755-771, 2012. 
[14] T. Mansour. Identities on harmonic and $q$-harmonic number sums. Afr. Mat., 23:135-143, 2012.

[15] T. Mansour, M. Shattuck, and C. Song. A q-analog of a general rational sum identity. Afr. Mat., 24:297-303, 2013.

[16] H. Prodinger. Some applications of the $q$-Rice formula. Random Struct. Alg., 19:552557, 2001.

[17] H. Prodinger. $q$-Identities of Fu and Lascoux proved by the $q$-Rice formula. Quaest. Math., 27:391-395, 2004.

[18] H. Prodinger. Identities involving rational sums by inversion and partial fraction decomposition. Appl. Anal. Discrete Math., 2:65-68, 2008.

[19] H. Prodinger. Identities involving harmonic numbers that are of interest for physicists. Utilitas Mathematica, 83:291-300, 2010.

[20] W. Szpankowski. Average case analysis of algorithms on sequences. John Wiley, New York, 2001.

[21] L. Van Hamme. Advanced Problem 6407. Amer. Math. Monthly, 40:703-704, 1982.

[22] W. Wang and C. Jia. Harmonic number identities via the Newton-Andrews method. Ramanujan J., in press.

[23] J. Zeng. On some q-identities related to divisor functions. Adv. Appl. Math., 34:313$315,2005$. 